\title{
Neurogranin as a cognitive biomarker in cerebrospinal fluid and blood exosomes for Alzheimer's disease and mild cognitive impairment
}

Weilin Liu' ${ }^{1,2}$, Huawei Lin ${ }^{1}$, Xiaojun $\mathrm{He}^{3}$, Lewen Chen ${ }^{3}$, Yaling Dai ${ }^{3}$, Weiwei $\mathrm{Jia}^{3}$, Xiehua Xue ${ }^{4}$, Jing Tao ${ }^{1}$ and Lidian Chen $^{3}$

\begin{abstract}
Alzheimer's disease (AD) is a progressive neurodegenerative disorder with clinical, biological, and pathological features occurring along a continuum from normal to end-stage disease. Currently, the diagnosis of AD depends on clinical assessments and post-mortem neuropathology, which is unbenefited early diagnosis and progressive monitoring. In recent years, clinical studies have reported that the level of cerebrospinal fluid (CSF) and blood neurogranin ( $\mathrm{Ng}$ ) are closely related to the occurrence and subsequent progression of $\mathrm{AD}$. Therefore, the study used meta-analysis to identify the CSF and blood Ng levels for the development of diagnosis biomarker of patients with $A D$ and mild cognitive impairment (MCl). We searched the Pubmed, Embase, Cochrane Library, and Web of Science databases. A total of 24 articles eligible for inclusion and exclusion criteria were assessed, including 4661 individuals, consisting of 1518 AD patients, $1501 \mathrm{MCl}$ patients, and 1642 healthy control subjects. The level of CSF Ng significantly increased in patients with $\mathrm{AD}$ and $\mathrm{MCl}$ compared with healthy control subjects (SMD: 0.84 [95\% Cl: 0.70-0.98], P< 0.001; SMD: 0.53 [95\% Cl: $0.40-0.66], P=0.008$ ), and higher in AD patients than in MCl patients (SMD: 0.18 [95\% Cl: 0.07-0.30], $P=0.002$ ), and CSF Ng level of patients with $\mathrm{MCl}-\mathrm{AD}$ who progressed from $\mathrm{MCl}$ to $\mathrm{AD}$ was significantly higher than that of patients with stable $\mathrm{MCl}$ (SMCI) (SMD: 0.71 [95\% Cl: 0.25-1.16], $P=0.002)$. Moreover, the concentration of $\mathrm{Ng}$ in blood plasma exosomes of patients with $\mathrm{AD}$ and $\mathrm{MCl}$ was lower than that of healthy control subjects (SMD: -6.657 [95\% Cl: -10.558 to -2.755$], P=0.001$; and SMD: -3.64 [95\% Cl: -6.50 to -0.78$], P=0.013)$, and which in patients with AD and $\mathrm{MCl}-\mathrm{AD}$ were also lower than those in patients with $\mathrm{SMCl}(P<0.001)$. Furthermore, regression analysis showed a negative relationship between MMSE scores and CSF Ng levels in MCl patients (slope $=-0.249$ [95\% Cl: -0.003 to $-0.495], P=0.047)$. Therefore, the Ng levels increased in CSF, but decreased in blood plasma exosomes of patients with $A D$ and $M C I-A D$, and highly associated with cognitive declines. These findings provide the clinical evidence that CSF and blood exosomes $\mathrm{Ng}$ can be used as a cognitive biomarker for AD and MCl-AD, and further studies are needed to define the specific range of $\mathrm{Ng}$ values for diagnosis at the different stages of $\mathrm{AD}$.
\end{abstract}

\section{Introduction}

Alzheimer's disease (AD) is a neurodegenerative disease with insidious and progressive onset of disease. It is also

\footnotetext{
Correspondence: Lidian Chen (cld@fjtcm.edu.cn)

${ }^{1}$ College of Rehabilitation Medicine, Fujian University of Traditional Chinese Medicine, Fuzhou, Fujian, China

2Department of Biochemistry and Molecular Biology, University of Oklahoma Health Sciences Center, Oklahoma City, OK, USA

Full list of author information is available at the end of the article

These authors contributed equally: Weilin Liu, Huawei Lin
}

one of the most common types of dementia and an important cause of death in the elderly, with more than $60 \%$ of all the dementia cases among elderly over 65 years old attributed to $\mathrm{AD}^{1}$. Currently, $\sim 35$ million people are suffering from $\mathrm{AD}$ or other types of dementia, and the number of dementia patients worldwide is predicted to reach 80 million by 2050 , with bringing enormous economic pressure to both the family and society ${ }^{2}$. As AD is clinically found to be relatively advanced, a large

\section{(c) The Author(s) 2020}

\footnotetext{
(c) Open Access This article is licensed under a Creative Commons Attribution 4.0 International License, which permits use, sharing, adaptation, distribution and reproduction cc) in any medium or format, as long as you give appropriate credit to the original author(s) and the source, provide a link to the Creative Commons license, and indicate if changes were made. The images or other third party material in this article are included in the article's Creative Commons license, unless indicated otherwise in a credit line to the material. If material is not included in the article's Creative Commons license and your intended use is not permitted by statutory regulation or exceeds the permitted use, you will need to obtain permission directly from the copyright holder. To view a copy of this license, visit http://creativecommons.org/licenses/by/4.0/.
} 
number of literatures have shown that the treatment of $\mathrm{AD}$ is poor ${ }^{3,4}$.

$\mathrm{AD}$ is characterized by progressive loss of memory and impairment of cognitive ability, but before showing typical symptoms of dementia, patients will have a pre-clinical mild cognitive impairment stage ${ }^{5}$. Mild cognitive impairment (MCI) is a condition in which an individual exhibits a slight decline in cognitive ability that is not perceived, and the early stage of $\mathrm{AD}$ does not impair the patient's ability of living and working, but if it is not prevented from developing in time, more than $50 \%$ of people with MCI will likely progress to dementia ${ }^{6}$. Therefore, there is an urgent need for cost-effective biomarkers for early diagnosis of AD.

Although the hallmark pathological features of $A D$, amyloid- $\beta(A \beta)$ deposition, phosphorylated Tau protein to form neurofibrillary tangles, and sustained neuroinflammatory reactions, have long been described in the brain $^{7-9}$. These important pathological changes lead to dysfunction and loss of neurons and synapses. Unfortunately, many intervention therapies targeting $A \beta$ and Tau have been unsuccessful ${ }^{10}$. Thus, we need further investigation on the etiology of $\mathrm{AD}$ and subsequent therapeutic intervention. In the past few years, accumulated evidence suggests that neurogranin $(\mathrm{Ng})$ as a post-synaptic protein is closely related to synaptic loss in AD patients ${ }^{11}$.

$\mathrm{Ng}$ is a neuron-specific and post-synaptic protein that binds to calmodulin and is abundantly expressed in the brain, particularly in the dendritic spine of hippocampus and cerebral cortex ${ }^{12}$. It is thought to be involved in synaptic plasticity and long-term potentiation (LTP) by regulating calcium-mediated signaling pathways ${ }^{13}$. There are many studies demonstrating that in animals, $\mathrm{Ng}$ knockdown models inhibit synaptic LTP and impact cognitive function ${ }^{13}$, whereas upregulation promotes LTP and improves cognition ${ }^{14}$. Similar findings also have been found that $\mathrm{Ng}$ is depleted in the brain, rose in cerebrospinal fluid (CSF), and associated with poorer cognitive performance in $\mathrm{AD}$ patients ${ }^{15} \mathrm{Ng}$ has abundant neuronal expression in the central nervous system and compared with traditional biomarkers, $\mathrm{Ng}$ can detect the pathological changes of $\mathrm{AD}$ at an earlier stage, even in the MCI stage, so far it is considered to be a potential biomarker for diagnosis of $\mathrm{AD}^{16}$. This study aimed to investigate $\mathrm{Ng}$ whether as a cognitive biomarker in the CSF and blood for diagnosis of AD and MCI based on high-level medical evidences. Therefore, we measured CSF and peripheral blood $\mathrm{Ng}$ levels in three groups of populations, healthy subjects, $\mathrm{MCI}$ patients, and $\mathrm{AD}$ patients, from cross-sectional and longitudinal studies.

\section{Methods}

The meta-analysis is conducted in strict accordance with the requirements of the Preferred Reporting Items for Systematic Reviews and Meta-analysis (PRISMA) statement ${ }^{17}$ and was registered at International Prospective Register of Systematic Reviews (https://www.crd. york.ac.uk/PROSPERO/) (numbers CRD42019135344 and CRD42019141393).

\section{Search strategy}

Two individuals independently searched all English articles using the Pubmed, Embase, Cochrane Library, and Web of Science databases through June 1, 2019. The search term was (Neurogranin OR NRGN OR Hng OR Protein Kinase C Substrate OR RC3) AND (Alzheimer's disease OR Mild cognitive impairment). The initial search generated 194 records from Pubmed, 708 records from Embase, and 17 records from the Cochrane Library, 527 records from the Web of Science database.

\section{Inclusion and exclusion criteria \\ Inclusion criteria}

The articles need to meet the following characteristics: (1) they must meet the diagnosis criteria of $\mathrm{AD}$ and $\mathrm{MCI}$ according with NINCDS-ADRDA Criteria ${ }^{18}$, or NIA-AA Criteria $^{19,20}$, or IWG-2 Criteria ${ }^{21}$. (2) The Ng concentration of CSF or blood must be one of the main variables of interest in patients with AD or MCI. (3) The reported data on $\mathrm{Ng}$ in at least two groups of subjects (AD, MCI, and healthy control subjects).

\section{Exclusion criteria}

First, we excluded the articles that were unrelated to Ng, $\mathrm{MCI}$, or $\mathrm{AD}$, or if they were review articles or animal experiments. In addition, studies were excluded that only reported the levels of $\mathrm{Ng}$ for participants with $\mathrm{MCI}$ or $\mathrm{AD}$ without control group. Thirdly, if articles did not have necessary $\mathrm{Ng}$ data, we also excluded them. In the end, we included 24 studies that fulfilled both the inclusion and exclusion criteria (Supplementary Fig. 1).

\section{Data extraction}

Two different individuals extracted the following data for the purposes of this meta-analysis. We extracted sample sizes, mean $\mathrm{Ng}$ concentrations or median, standard deviations or interquartile range, and we regarded them as the primary outcome. In addition, data on the author and publication year of the article, the average age of the patient, the gender distribution, the Mini-Mental State Examination (MMSE) score, the sample source (CSF or blood or serum or plasma or exosomes), and the assay type were also extracted.

\section{Quality assessment}

We used a checklist of Cross-sectional Study/Prevalence Quality to assess the methodological quality of the studies which was recommended by Agency for 
Healthcare Research and Quality (AHRQ), and AHRQ checklist consists of 11 items $^{22}$ (https://www.ncbi.nlm. nih.gov/books/NBK35156/). The 11-item ranges from 0 to 8 stars for cross-sectional studies. If an item was answered 'NO' or 'UNCLEAR', it would be scored ' 0 ', if it was answered 'YES', then the item scored ' 1 '. Article quality was evaluated as follows: low quality $=0-3$; moderate quality $=4-7$; high quality $=8-11$.

In addition to the longitudinal observational study, we chose the Newcastle-Ottawa Scale (NOS) that is recommended by the Cochrane Collaboration ${ }^{23}$. The NOS ranges from 0 to 9 stars for case-control studies and cohort studies. A study can be awarded a maximum score of 1 star for each numbered item within the Selection and Exposure categories. A maximum of 2 stars can be given for comparability. A score above 5 implies that the study has high quality.

\section{Statistical analysis}

We used the Stata 14.0 (Stata Corp) software to perform all statistical analyses. For certain studies with only median and interquartile ranges available in the articles, we need to estimates the means and standard deviations according to $\mathrm{Hozo}^{24}$ and Dehui Luo ${ }^{25}$. Then, we calculated standardized mean difference (SMD) and 95\% confidence intervals and generated a forest plots to compare the mean $\mathrm{Ng}$ levels between $\mathrm{AD}$ or $\mathrm{MCI}$ and healthy control in order to eliminate the effects between different measurement scales. The random-effects model was chosen because we assumed heterogeneity between the articles. In the next statistical analysis, first, we performed overall meta-analysis between AD vs. healthy control, $\mathrm{MCI}$ vs. healthy control, and $\mathrm{AD}$ vs. MCI. Whether there is heterogeneity between the articles was evaluated by $Q$ test, and the statistical significance was set to $P$-value $<$ 0.1 . We further used the $I^{2}$ index to evaluate the influence of heterogeneity, $I^{2}$ was $0.25,0.50$, and 0.75 , which suggested small, moderate and high levels of heterogeneity ${ }^{26}$, respectively. Secondly, we conducted a subgroup metaanalysis study to test whether there was in significant difference in age-matched or mismatched groups, analytical method for Ng, different MMSE groups ( $\geq 20$ and $<20$ ) and type of study. Thirdly, we performed a series of meta-regression analyses to assess the effect of multiple regulatory variables on SMD. By estimating the impact of a single study on the results of the meta-analysis, sensitivity analysis was also performed to test the robustness of the results. Then, we generated a funnel plot and chosen Egger test to evaluate the potential for publication bias for each meta-analysis. For publication bias, we adopted the trim and fill method to supplement the missing articles and verify if the results were robust by comparing them with the results before supplementing. All statistical significances were set at $P$-value $<0.05$ except where noted, $P$-value below 0.1 was reported as a trend.

\section{Results}

\section{Search results}

According to our search strategy, we obtained a total of 1446 articles from four databases. Among them, we removed the duplicated 292 articles. By screening the titles and abstracts, we removed 1119 articles that were not related to the topic or reviews and animal experiments, and then according to our inclusion and exclusion criteria, we removed 11 more articles for the following reasons: lack of necessary $\mathrm{Ng}$ data, articles without healthy controls and date on brain of tissues instead of CSF, and finally, we received 24 articles that met the inclusion criteria $^{27-50}$. The Flow Diagram showed the detailed process of selection (Supplementary Fig. S1). The characteristics of the 24 included studies are shown, including 4661 individuals, consisting of 1518 AD patients, 1501 MCI patients, and 1642 healthy control subjects (see Supplementary Table S1 for details).

\section{Quality assessment}

A total of 17 cross-sectional and 7 longitudinal studies were included in this review. We evaluated the quality of these articles according to the corresponding scales (Supplementary Tables S2 and S3). The results show that the scores of articles assessed by 11-item ranged from 4 to 8 (mean $=6.2$ ) and all of articles are in the moderate quality range, while the studies evaluated by the NOS ranged from 3 to 7 (mean $=5.7$ ) and the overall quality of the literature is higher.

\section{Association of cerebrospinal fluid $\mathrm{Ng}$ with cognitive impairment in patients with $A D$ and $\mathrm{MCl}$}

First, we compared CSF Ng levels between AD patients and $\mathrm{HC}$ subjects, extracting data from 2891 individuals in 19 studies $^{27,28,31,32,34-48}$. Random-effects meta-analysis was performed, and the results showed that CSF Ng levels were significantly elevated in AD patients compared with healthy control subjects (SMD: 0.84 [95\% CI: 0.70-0.98], $z=11.72, P<0.001$, Fig. 1 and Supplementary Table S4). However, high heterogeneity between studies was observed $\left(Q=36.19\right.$, df. $=18, I^{2}=59.0, P=0.001$, Supplementary Table $\mathrm{S} 4$ ), and sensitivity analysis indicated that our results were not over-affected by a particular study.

Then, we compared the CSF Ng levels between MCI and healthy control, including data of 2203 individuals from 15 studies ${ }^{27,28,30-34,36,38-41,43,45,46}$. The results showed that CSF Ng levels were significantly higher in MCI patients compared with healthy control subjects (SMD: 0.5 [95\% CI: 0.39-0.6], $\mathrm{z}=7.29, P<0.001$, Fig. 2 and Supplementary Table S4). There was moderate heterogeneity between studies $\left(Q=30.83\right.$, df. $=14, I^{2}=54.6$, $P=0.0064$, Supplementary Table S4), and sensitivity analysis showed the conclusions were not affected. 


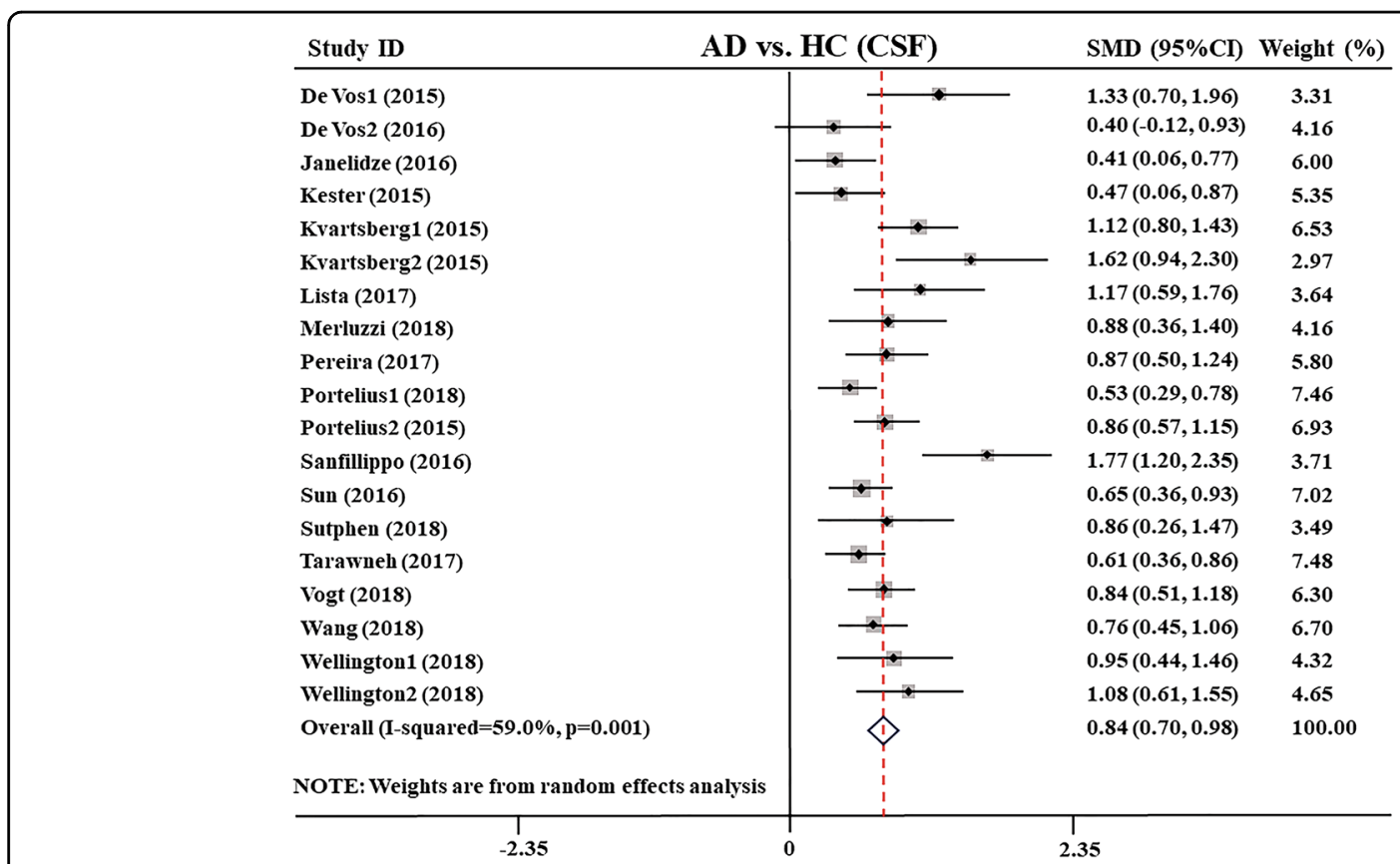

Fig. 1 Forest plot of random-effects meta-analysis of cerebrospinal fluid $\mathrm{Ng}$ levels between AD patients and HC subjects. Data include 2891 individuals from 19 studies. The squares indicate individual study SMD and their corresponding 95\% Cls and the sizes of the squares are proportional to study weight. Ng neurogranin, SMD standard mean difference, Cl confidence interval, AD Alzheimer's disease, HC healthy control.

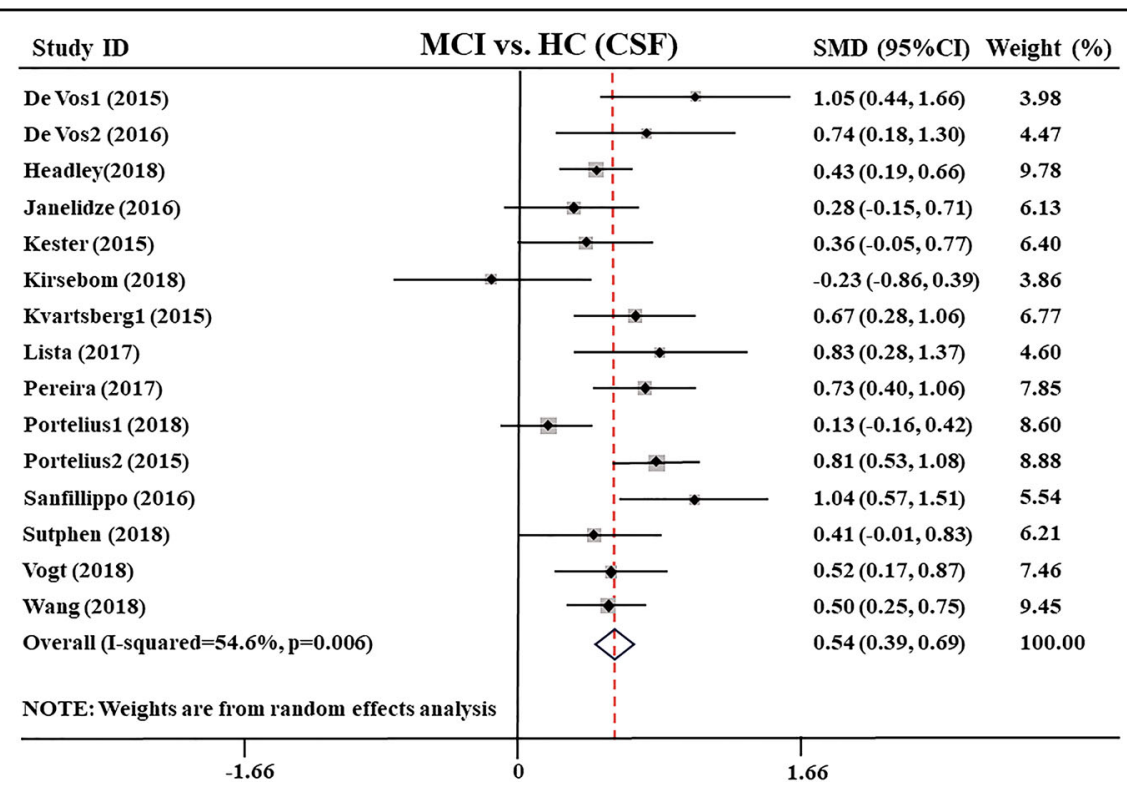

Fig. 2 Forest plot of random-effects meta-analysis of cerebrospinal fluid $\mathrm{Ng}$ levels between $\mathrm{MCl}$ patients and $\mathrm{HC}$ subjects. Data include 2203 individuals from 15 studies. The squares indicate individual study SMD and their corresponding 95\% Cls and the sizes of the squares are proportional to study weight. Ng neurogranin, SMD standard mean difference, $\mathrm{Cl}$ confidence interval, $\mathrm{MCl}$ mild cognitive impairment, HC healthy control.

Moreover, the distinctions between $\mathrm{AD}$ and $\mathrm{MCI}$ in CSF $\mathrm{Ng}$ levels, extracted from 14 studies $^{27,28,31,32,34,36,38-43,45,46}$ encompassing a sample of 2216 individuals, were also tested. The comparison showed that the CSF Ng level in the
AD group was higher than that in the MCI group (SMD: 0.18 [95\% CI: 0.07-0.30], $z=3.05, P=0.002$, Fig. 3 and Supplementary Table S4). The heterogeneity between the studies was low $\left(Q=20.44\right.$, df. $=13, I^{2}=36.4, P=0.085$, 


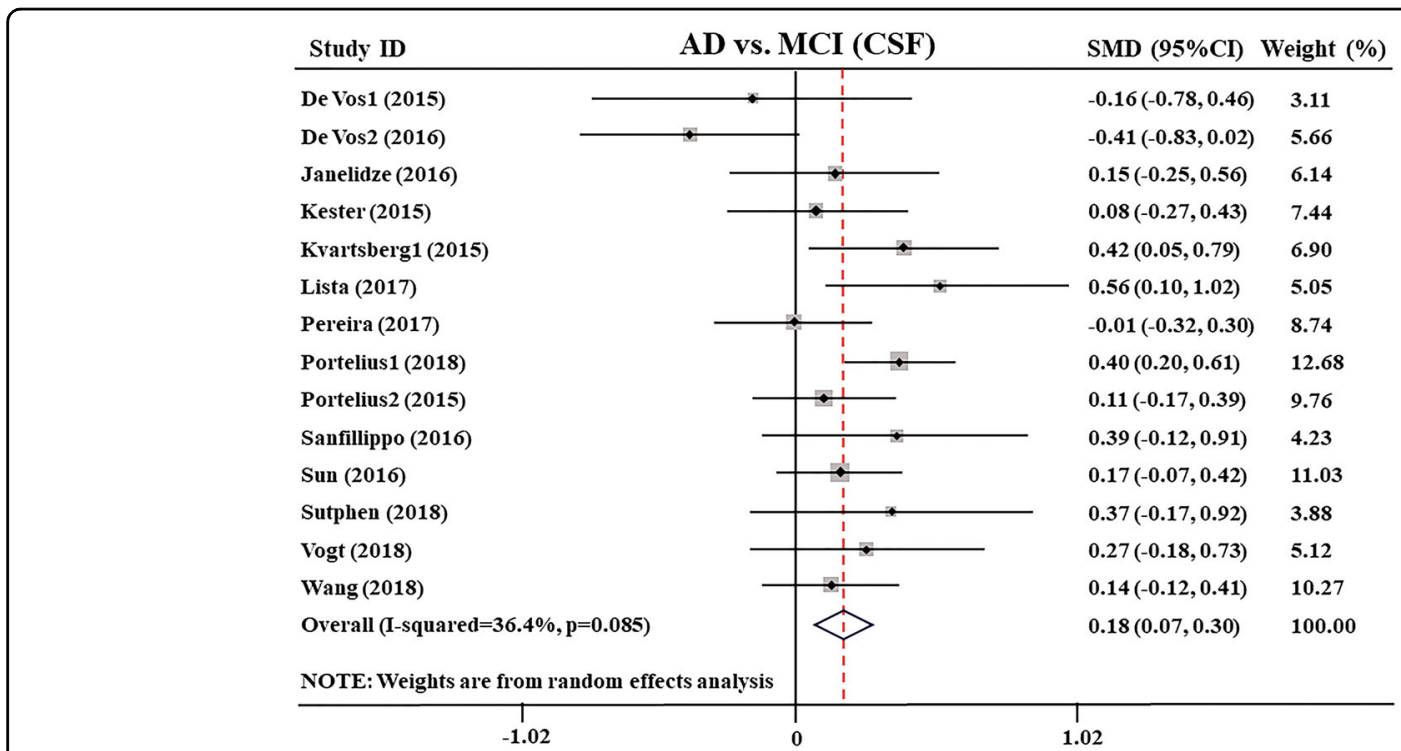

Fig. 3 Forest plot of random-effects meta-analysis of cerebrospinal fluid $\mathbf{~ N g}$ levels between AD and $\mathbf{M C l}$ patients. Data include 2216 individuals from 14 studies. The squares indicate individual study SMD and their corresponding 95\% Cls and the sizes of the squares are proportional to study weight. $\mathrm{Ng}$ neurogranin, SMD standard mean difference, $\mathrm{Cl}$ confidence interval, AD Alzheimer's disease, MCI mild cognitive impairment.

\begin{tabular}{|c|c|c|c|}
\hline \multicolumn{2}{|c|}{ MCI-AD vs. sMCI (CSF) } & SMD $(95 \% C I)$ & Weight (\%) \\
\hline Janelidze (2016) & $\frac{1}{1}$ & $0.36(-0.06,0.78)$ & 28.11 \\
\hline Kester (2015) & & $0.79(0.19,1.38)$ & 22.53 \\
\hline Kvartsberg1 (2015) & 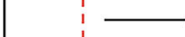 & $1.68(0.91,2.46)$ & 17.84 \\
\hline Portelius2 (2015) & & $0.41(0.10,0.72)$ & 31.51 \\
\hline Overall (I-squared $=71.3 \%, p=0.015$ ) & & $0.71(0.25,1.16)$ & 100.00 \\
\hline NOTE: Weights are from random effects analysis & 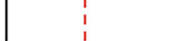 & & \\
\hline$\frac{1}{-2.46}$ & $T_{0}$ & $\begin{array}{c}1 \\
2.46\end{array}$ & \\
\hline
\end{tabular}

Fig. 4 Forest plot of random-effects meta-analysis of CSF neurogranin levels between $\mathrm{MCl}-\mathrm{AD}$ and sMCI patients. Data include 360 individuals from four studies. The squares indicate individual study SMD and their corresponding 95\% Cls and the sizes of the squares are proportional to study weight. MCl-AD mild cognitive impairment patients who progressed to Alzheimer's disease, sMCl stable mild cognitive impairment, CSF cerebrospinal fluid, SMD standard mean difference, Cl confidence interval.

Supplementary Table S4) and was not significantly affected by the specific study.

In addition, from four studies ${ }^{31,32,34,40}$, the researchers conducted follow-up of MCI patients for different lengths of time, and measured the CSF $\mathrm{Ng}$ level of patients with MCI-AD who progressed from MCI to AD. The results showed that the CSF $\mathrm{Ng}$ level of patients with MCI-AD was significantly higher than that of patients with stable MCI (sMCI) (SMD: 0.71 [95\% CI: 0.25-1.16], $z=3.06, P=0.002$, Fig. 4 and Supplementary Table S4).

Furthermore, meta-regression analysis result showed a negative relationship (slope $=-0.249$ [95\% CI: -0.003 to $-0.495], P=0.047$, Supplementary Fig. S2 and Supplementary Table S5) between the MMSE scores and effect size in MCI patients, indicating that the lower the MMSE scores, the SMD increased implying larger CSF Ng levels compared with healthy control subjects.

\section{Association of blood $\mathrm{Ng}$ with cognitive impairment in patients with $A D$ and $\mathrm{MCl}$}

We found that blood plasma $\mathrm{Ng}$ levels were determined for $\mathrm{AD}$ in two studies ${ }^{27,35}$. Our meta-analysis results showed that there was no statistical difference in plasma $\mathrm{Ng}$ levels between $\mathrm{AD}$ patients with healthy control subjects (SMD: -0.252 [95\% CI: -0.663 to 0.1590], $z=$ $1.20, P=0.230$, Supplementary Fig. S3), which was consistent with their respective findings.

However, in blood exosomes, meta-analysis results from two other studies ${ }^{29,49}$ showed that compared with the healthy control subjects, the levels of blood plasma neuronally derived exosome (NDE) $\mathrm{Ng}$ in $\mathrm{AD}$ patients have 


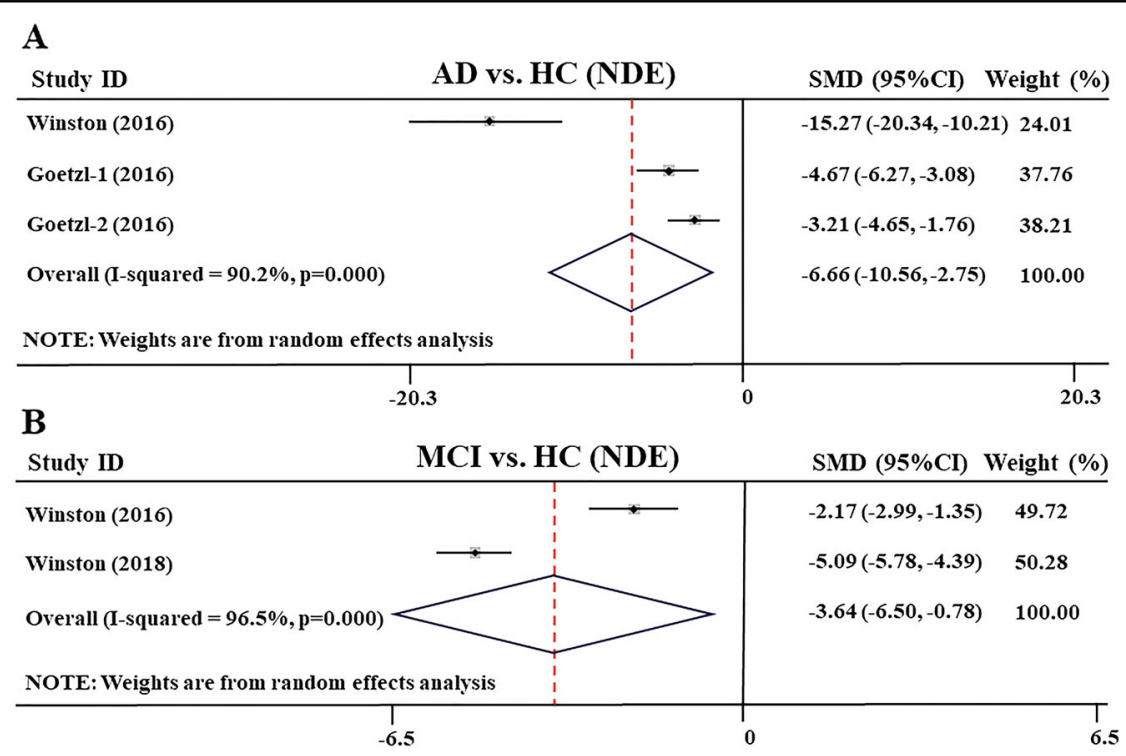

Fig. 5 Forest plot of random-effects meta-analysis of blood plasma neuronal derived exosomes $\mathrm{Ng}$ levels between AD, MCI patients and HC subjects. a Data include 62 individuals from two studies (Goetzl's study reports results from two groups of AD patients and HC subjects) for meta-analysis of exosomes $\mathrm{Ng}$ levels between $\mathrm{AD}$ and $\mathrm{HC}$. $\mathbf{b}$ Data include 187 individuals from two studies for meta-analysis of exosomes $\mathrm{Ng}$ levels between $\mathrm{MCl}$ and $\mathrm{HC}$. The squares indicate individual study SMD and their corresponding 95\% $\mathrm{Cls}$ and the sizes of the squares are proportional to study weight. SMD standard mean difference, $\mathrm{Cl}$ confidence interval, AD Alzheimer's disease, MCI mild cognitive impairment, $\mathrm{HC}$ healthy control.

an obviously decrease (SMD: -6.657 [95\% CI: -10.558 to $-2.755], z=3.34, P=0.001$, Fig. 5a). In addition, Abner et al. ${ }^{51}$ reported that the concentration of blood plasma NDE Ng was reduced in older cognitively intact subjects tested by two samples collected at 3- and 11-year intervals $(285.1 \pm 76.8 \mathrm{pg} / \mathrm{mL} \quad$ vs. $224.8 \pm 61.0 \mathrm{pg} / \mathrm{mL}, \quad P<0.05)$, especially those subjects who transitioned to $\mathrm{AD}$ that the level of NDE Ng decreased significantly. These results were similar to the decreased $\mathrm{Ng}$ levels seen in human brain tissue of $\mathrm{AD}$ patients ${ }^{52}$.

Moreover, from two studies ${ }^{49,50}$, in blood exosomes, meta-analysis showed that the levels of blood plasma NDE $\mathrm{Ng}$ were significantly lower in MCI patients than in healthy control subjects (SMD: -3.64 [95\% CI: -6.50 to -0.78 ] $z=2.49, P=0.013$, Fig. 5b). In addition, a study reported that the blood plasma NDE Ng in patients with $\mathrm{MCI}-\mathrm{AD}$ and $\mathrm{AD}$ were also significantly lower than those in sMCI patients $(P<0.001)^{49}$.

\section{Investigation of heterogeneity}

To identify the cause of high heterogeneity found in the comparison of CSF Ng levels between AD and healthy control, we performed subgroup analyses, and the results showed that the age-matched and age-mismatched, the MMSE $\geq 20$ and MMSE $<20$, the Ng ELISA and MSD analytical methods did not affect the heterogeneity (Supplementary Table S6 and Supplementary Figs. S4-S6). But by comparing the types of studies, we found crosssectional studies have higher heterogeneity $\left(I^{2}=65 \%\right.$,
$P<0.001$, Supplementary Table S6 and Supplementary Fig. S7), while longitudinal studies have the opposite $\left(I^{2}=0\right.$, $P=0.515$, Supplementary Table S6 and Supplementary Fig. S7). And we performed a series of meta-regression analyses and the results showed that age, gender, and MMSE scores could not be regarded as effect factors to explain the heterogeneity between $\mathrm{AD}$ and healthy control ( $P>0.05$ for all the analyses).

Similarly, we found the comparison of CSF Ng levels between $\mathrm{MCI}$ and healthy control, age matching does not affect heterogeneity (Supplementary Table S6 and Supplementary Fig. S8), and cross-sectional studies have higher heterogeneity $\left(I^{2}=60.5 \%, P=0.005\right.$, Supplementary Table S6 and Supplementary Fig. S9), but longitudinal studies lower $\left(I^{2}=33.8 \%, P=0.196\right)$. Then, in the subgroup analysis of analytical methods, we found that the MSD analytical method has no heterogeneity $\left(I^{2}=\right.$ $43.7 \%, P=0.149$, Supplementary Table S6 and Supplementary Fig. S10), and the ELISA analytical method has higher heterogeneity $\left(I^{2}=66.1 \%, P=0.001\right.$, Supplementary Table S6 and Supplementary Fig. S10). Therefore, the type of studies and $\mathrm{Ng}$ diagnostic method are both important sources of heterogeneity regarding CSF Ng levels in patients with $\mathrm{AD}$ and $\mathrm{MCI}$.

\section{Publication bias}

We used funnel plot to make a preliminary judgment, and then used the Egger's test to confirm that there were no publication bias for $\mathrm{Ng}$ level comparisons between $\mathrm{AD}$ 
and healthy control, $\mathrm{MCI}$ and healthy control, $\mathrm{AD}$ and MCI $(P=0.156, P=0.110, P=0.156$, Supplementary Figs. S11-S13).

\section{Discussion}

Recently, the study of Ng levels in the CSF and blood of patients with $\mathrm{AD}$ is a hot topic. In this study, we used meta-analysis to identify the CSF and blood Ng levels for the development of diagnosis biomarkers of patients with $\mathrm{AD}$ and mild cognitive impairment (MCI). We extracted a total of 4661 individuals from 24 studies for this metaanalysis and obtained two important findings. Firstly, the level of $\mathrm{Ng}$ in CSF of patients with $\mathrm{AD}$ and $\mathrm{MCI}$ increased, simultaneously, there was higher in patients with $\mathrm{AD}$ and MCI-AD than in sMCI patients. And CSF Ng level increased with cognitive declines and negatively associated with MMSE scores. Secondly, the concentration of $\mathrm{Ng}$ in blood plasma exosomes of patients with $\mathrm{AD}$ and $\mathrm{MCI}$ decreased, and $\mathrm{Ng}$ in blood plasma exosomes of patients with $\mathrm{AD}$ and $\mathrm{MCI}-\mathrm{AD}$ were also lower than those in patients with sMCI. Therefore, these findings reinforce the clinical evidence that CSF and blood exosomes $\mathrm{Ng}$ can be used as a cognitive biomarker for $\mathrm{AD}$ and MCI.

For traditional biomarkers, $A \beta$ and Tau are used as diagnostic markers for $\mathrm{AD}$ and $\mathrm{MCI}^{53,54}$. The former is caused by incorrect cleavage of the amyloid precursor protein, resulting in the aggregation of $A \beta$ monomers into misfolded $A \beta$ oligomers, which can inhibit the glutamate reuptake of neurons and lead to excessive activation of neurons, thereby exerting its neurotoxic effect ${ }^{55}$. In addition, Tau is the main neuronal microtubule assembly protein. In the brain of patients with $\mathrm{AD}$, Tau is abnormally hyperphosphorylated and aggregated into paired helical filaments, which appear as which manifest as neurofibrillary tangles (NFTs) ${ }^{56}$. In the past few years, emerging new biomarkers for early diagnosis of $\mathrm{AD}$ have been found, among which, the level of Ng in CSF of AD patients is significantly positively correlated with CSF total Tau and phosphorylated $\operatorname{tau}^{27,31,32,41,47,48}$, but the reports of the relationship with $A \beta$ is inconsistent. There was a positive correlation with $A \beta 40^{31}$ and a negative correlation with $A \beta 1-42 / A \beta 1-40^{27}$, and also reported that there is no correlation between $\mathrm{Ng}$ and $\mathrm{A} \beta 42^{32,41}$. Unlike the pathological mechanism of $\mathrm{A} \beta$ and $\mathrm{Tau}, \mathrm{Ng}$ responds to synaptic loss or synaptic plasticity disorder in the process of $\mathrm{AD}$, and synaptic integration mirrored by the increase of CSF $\mathrm{Ng}$ level may occur earlier than the structural degradation represented by Tau pathology ${ }^{57,58}$. There is considered that synaptic dysfunction and degeneration are a central event in AD pathology from the beginning of the early stages of $\mathrm{AD}$, because learning and memory are formed through synaptic plasticity ${ }^{56,57}$. Comparing with extracellular plaques and neurofibrillary tangles, synaptic loss is directly associated with the degree of dementia, especially hippocampal synaptic functional damage, closely related to memory impairments ${ }^{59,60}$. Therefore, $\mathrm{Ng}$ as a post-synaptic membrane protein can be a promising tool for early diagnosis of cognitive decline. $\mathrm{Ng}$ binding to calmodulin weakened when the synaptic structure is disrupted, affecting the transmission of $\mathrm{Ca}^{2+}$ between the synapses and the formation of LTP, thus it leads to early cognitive decline ${ }^{61}$. And more importantly, studies in humans have shown that the expression of $\mathrm{Ng}$ was decreased in the frontal and parietal cortices of $\mathrm{AD}$ patients, and $\mathrm{Ng}$ was significantly associated with the degree of amyloid and Tau pathology ${ }^{52,62}$. Here, we analyzed follow-up data from $360 \mathrm{MCI}$ patients and showed that the level of $\mathrm{Ng}$ in CSF of patients with MCI-AD were obviously higher than that of patients with $\mathrm{sMCI}^{31,32,34,40}$. In addition, a meta-analysis also showed that CSF Ng levels are higher in AD and MCI compared to health subjects, and higher in $\mathrm{AD}$ compared to $\mathrm{MCI}^{63}$. They included 16 articles to clarify whether CSF Ng can be a reliable diagnosis for $\mathrm{AD}$ and $\mathrm{MCI}$, and we included 24 articles, which is a larger patient size to analyze the CSF Ng for the diagnosis of AD and MCI. Importantly, we did a series of subgroup analysis and regression analysis to confirm the conclusion. Therefore, the CSF Ng may have the potential to reflect synaptic degeneration at the early stage and can be served as a prognostic cognitive biomarker for MCI-AD and AD.

Although CSF biomarkers are highly accurate in the diagnosis of $\mathrm{AD}$ and $\mathrm{MCI}$, they are more traumatic to patients. However, using peripheral blood biomarkers for $\mathrm{AD}$ diagnosis has the advantages of less trauma, easy sampling, and widespread adoption, but the sensitivity is lower, thus how to find more sensitive biomarkers in peripheral blood has become a key problem to be solved. The blood plasma neuronally derived exosome provides new insights into reflecting the pathogenesis of $\mathrm{AD}^{64}$. Interestingly, we found that, contrary to the level of $\mathrm{Ng}$ in $\mathrm{CSF}$, the level of $\mathrm{Ng}$ in blood plasma neuronally derived exosomes was decreased in patients with $\mathrm{AD}$ and $\mathrm{MCI}-$ $\mathrm{AD}$, and there was no change in blood plasma $\mathrm{Ng}$ of $\mathrm{AD}$ patients. Therefore, the concentration of $\mathrm{Ng}$ in the blood plasma exosome was the same as that in the brain tissues of AD patients ${ }^{52,62}$, but the level in CSF was the opposite. At present, there is no specific answer to explain this result. There are literatures showing that 39 endogenous $\mathrm{Ng}$ peptides have been identified by combining hybrid immunoaffinity and high-resolution mass spectrometry in CSF of patients with $\mathrm{AD}$, and full-length $\mathrm{Ng}$ was modified including disulfide bridges or glutathione ${ }^{62}$. Of which Ng48-76 peptide showed the most significant increase in CSF of patients with AD compared to the healthy control subjects, importantly, Ng48-76 is also the major peptide in $\mathrm{AD}$ brain tissue, but this peptide was not found in plasma $^{34}$. Therefore, we speculate that the increase of $\mathrm{Ng}$ 
level in CSF and the decrease of $\mathrm{Ng}$ in blood plasma exosomes and brain tissues in patients with $\mathrm{AD}$ and MCI$\mathrm{AD}$ are related with the decomposition of $\mathrm{Ng}$ into many peptides modified by disulfide bridges or glutathione, and releasing into CSF. This leads to abnormal synapses or even death of synapses and the secretion of synapsesderived exosomes are unavailable. In the future, the sensitivity and accuracy of AD and MCI-AD diagnosis may be improved if the Ng specific peptides can be found in plasma exosomes, and calculate its ratios of peptides to the full length of Ng in CSF and blood exosomes. Recent study reported that the levels of $A \beta 42$, T-tau, and P-T181tau in CSF were consistent with those of blood exosomes $\mathrm{A} \beta 42, \mathrm{~T}-\mathrm{tau}$, and P-T181-tau ${ }^{65}$. These indicate that the change trend of $\mathrm{Ng}$ and $\mathrm{A} \beta 1-42$ in peripheral blood exosomes is opposite. If they can be detected at the same time, it may be more sensitive to the diagnosis of $\mathrm{AD}$ and MCI. In addition, Janelidze et al. ${ }^{31}$ showed that the CSF $\mathrm{Ng}$ was higher in patients with chronic ischemic vascular dementia (VD) compared with healthy control subjects. Therefore, Ng also may be considered as a prognostic cognitive biomarker for VD.

Taken together, these findings reinforce the clinical evidence that CSF and blood exosomes $\mathrm{Ng}$ can be used as a cognitive biomarker for $\mathrm{AD}$ and $\mathrm{MCI}-\mathrm{AD}$, but it is still difficult to identify the different stages of the disease through the specific range of $\mathrm{Ng}$ values, therefore, further studies are needed to define the $\mathrm{Ng}$ values for diagnosis of MCI-AD and AD. In addition, with the development of $\mathrm{AD}$ diagnostic criteria, the latest research framework focuses on the diagnosis of $\mathrm{AD}$ and $\mathrm{MCI}$ using biomarkers, including beta amyloid deposition, pathological tau and neurodegeneration, called AT $(\mathrm{N})$ biomarker system $^{66}$, and the system accepts novel biomarkers added to existing frameworks. Therefore, in the future, the integration of traditional and novel biomarkers of blood exosomes and CSF can be used as a more sensitive and accurate biomarkers system for the diagnosis of $\mathrm{AD}$ and MCI.

\section{Limitations}

At the beginning of the meta-analysis, we wanted to extract data from all the articles related to the topic, but after careful scrutiny, we had to exclude some studies that did not have the necessary data. And the funnel plot showed that there were many small sample sizes and lowquality studies. Supplementation of the missing study by the trim and fill method shifted the center line of the funnel plot to the right, which may affect the robustness of the results.

In addition, we found a higher heterogeneity in the comparison of $\mathrm{AD}$ patients with healthy controls and $\mathrm{MCI}$ patients with healthy controls. The high heterogeneity of the meta-analysis is being mainly due to the type of study and $\mathrm{Ng}$ diagnostic method. Thus, in the future, we should adopt longitudinal tracking and appropriate analytical method for diagnosis of $\mathrm{Ng}$ in $\mathrm{AD}$ and MCI-AD patients.

\section{Acknowledgements}

This study was supported by grants from the National Natural Science Foundation of China (Grant Numbers 81774424 and 81873353).

\section{Author details}

${ }^{1}$ College of Rehabilitation Medicine, Fujian University of Traditional Chinese Medicine, Fuzhou, Fujian, China. '2Department of Biochemistry and Molecular Biology, University of Oklahoma Health Sciences Center, Oklahoma City, OK, USA. ${ }^{3}$ The Academy of Rehabilitation Industry, Fujian University of Traditional Chinese Medicine, Fuzhou, Fujian, China. ${ }^{4}$ Affiliated Rehabilitation Hospital, Fujian University of Traditional Chinese Medicine, Fuzhou, Fujian, China

Conflict of interest

The authors declare that they have no conflict of interest.

\section{Publisher's note}

Springer Nature remains neutral with regard to jurisdictional claims in published maps and institutional affiliations.

Supplementary Information accompanies this paper at (https://doi.org/ 10.1038/s41398-020-0801-2).

Received: 2 December 2019 Revised: 12 March 2020 Accepted: 25 March 2020

Published online: 29 April 2020

\section{References}

1. Alzheimer's Association. 2018 Alzheimer's disease facts and figures. Alzheimers Dement. 14, 367-429 (2018).

2. Querfurth, H. W. \& LaFerla, F. M. Alzheimer's disease. N. Engl. J. Med. 362 , 329-344 (2010).

3. Egan, M. F. et al. Randomized trial of verubecestat for mild-to-moderate Alzheimer's disease. N. Engl. J. Med. 378, 1691-1703 (2018).

4. Egan, M. F. et al. Randomized trial of verubecestat for prodromal Alzheimer's disease. N. Engl. J. Med. 380, 1408-1420 (2019).

5. Petersen, R. C. et al. Mild cognitive impairment as a diagnostic entity. J. Intern. Med. 256, 183-194 (2004).

6. Behrman, S., Valkanova, V. \& Allan, C. L. Diagnosing and managing mild cognitive impairment. Practitioner 261, 17-20 (2017).

7. Hardy, J. \& Selkoe, D. J. The amyloid hypothesis of Alzheimer's disease: progress and problems on the road to therapeutics. Science 297, 353-356 (2002).

8. Ballatore, C., Lee, V. M. \& Trojanowski, J. Q. Tau-mediated neurodegeneration in Alzheimer's disease and related disorders. Nat. Rev. Neurosci. 8, 663-672 (2007)

9. Kinney, J. W. et al. Inflammation as a central mechanism in Alzheimer's disease. Alzheimers Dement. 4, 575-590 (2018).

10. Honig, L. S. et al. Trial of Solanezumab for mild dementia due to Alzheimer's disease. N. Engl. J. Med. 378, 321-330 (2018).

11. Gerendasy, D. D. \& Sutcliffe, J. G. RC3/neurogranin, a postsynaptic calpacitin for setting the response threshold to calcium influxes. Mol. Neurobiol. 15, 131-163 (1997).

12. Pak, J. H. et al. Involvement of neurogranin in the modulation of calcium/ calmodulin-dependent protein kinase II, synaptic plasticity, and spatial learning: a study with knockout mice. Proc. Natl Acad. Sci. USA 97, 11232-11237 (2000).

13. Huang, K. P. et al. Neurogranin/RC3 enhances long-term potentiation and learning by promoting calcium-mediated signaling. J. Neurosci. 24, 10660-10669 (2004).

14. Hayashi, Y. Long-term potentiation: two pathways meet at neurogranin. EMBO J. 28, 2859-2860 (2009).

15. Thorsell, A. et al. Neurogranin in cerebrospinal fluid as a marker of synaptic degeneration in Alzheimer's disease. Brain Res. 1362, 13-22 (2010). 
16. Laterza, O. F. et al. Identification of novel brain biomarkers. Ciln. Chem. 52, 1713-1721 (2006).

17. Moher, D., Liberati, A., Tetzlaff, J. \& Altman, D. G., PRISMA, Group. Preferred reporting items for systematic reviews and meta-analyses: the PRISMA statement. BMJ 339, b2535 (2009).

18. Dubois, B. et al. Research criteria for the diagnosis of Alzheimer's disease: revising the NINCDS-ADRDA criteria. Lancet Neurol. 6, 734-746 (2007).

19. McKhann, G. M. et al. The diagnosis of dementia due to Alzheimer's disease: recommendations from the National Institute on Aging-Alzheimer's association workgroups on diagnostic guidelines for Alzheimer's disease. Alzheimers Dement. 7, 263-269 (2011).

20. Albert, M. S. et al. The diagnosis of mild cognitive impairment due to Alzheimer's disease: recommendations from the National Institute on AgingAlzheimer's Association workgroups on diagnostic guidelines for Alzheimer's disease. Alzheimers Dement. 7, 270-279 (2011).

21. Dubois, B. et al. Advancing research diagnostic criteria for Alzheimer's disease: the IWG-2 criteria. Lancet Neurol. 13, 614-629 (2014).

22. Zeng, $X$. et al. The methodological quality assessment tools for preclinical and clinical studies, systematic review and meta-analysis, and clinical practice guideline: a systematic review. J. Evid. Based Med. 8, 2-10 (2015).

23. Higgins, J. P. \& Green, S. (eds) Cochrane Handbook for Systematic Reviews of Interventions Version 5.1.0. (Cochrane, 2013).

24. Hozo, S. P., Djulbegovic, B. \& Hozo, I. Estimating the mean and variance from the median, range, and the size of a sample. BMC Med. Res. Methodol. 5, 13 (2005).

25. Luo, D., Wan, X., Liu, J. \& Tong, T. Optimally estimating the sample mean from the sample size, median, mid-range, and/or mid-quartile range. Stat. Methods Med. Res. 27, 1785-1805 (2018).

26. Higgins, J. P., Thompson, S. G., Deeks, J. J. \& Altman, D. G. Measuring inconsistency in meta-analyses. BMJ 327, 557-560 (2003).

27. De Vos, A. et al. C-terminal neurogranin is increased in cerebrospinal fluid but unchanged in plasma in Alzheimer's disease. Alzheimers Dement. 11, 1461-1469 (2015).

28. De Vos, A. et al. The cerebrospinal fluid neurogranin/BACE1 ratio is a potential correlate of cognitive decline in Alzheimer's disease. J. Alzheimers Dis. 53 1523-1538 (2016).

29. Goetzl, E. J. et al. Decreased synaptic proteins in neuronal exosomes of frontotemporal dementia and Alzheimer's disease. FASEB J. 30, 4141-4148 (2016).

30. Headley, A. et al. Neurogranin as a predictor of memory and executive function decline in MCl patients. Neurology 90, e887-e895 (2018).

31. Janelidze, S. et al. Cerebrospinal fluid neurogranin and YKL-40 as biomarkers of Alzheimer's disease. Ann. Clin. Transl. Neurol. 3, 12-20 (2016).

32. Kester, M. I. et al. Neurogranin as a cerebrospinal fluid biomarker for synaptic loss in symptomatic Alzheimer disease. JAMA Neurol. 72, 1275-1280 (2015).

33. Kirsebom, B. E. et al. Cerebrospinal fluid neurogranin/ $\beta$-site APP-cleaving enzyme 1 predicts cognitive decline in preclinical Alzheimer's disease. Alzheimers Dement. 4, 617-627 (2018).

34. Kvartsberg, $H$. et al. Cerebrospinal fluid levels of the synaptic protein neurogranin correlates with cognitive decline in prodromal Alzheimer's disease. Alzheimers Dement. 11, 1180-1190 (2015).

35. Kvartsberg, $\mathrm{H}$. et al. Characterization of the postsynaptic protein neurogranin in paired cerebrospinal fluid and plasma samples from Alzheimer's disease patients and healthy controls. Alzheimers Res. Ther. 7, 40 (2015).

36. Lista, S. et al. Cerebrospinal fluid neurogranin as a biomarker of neurodegenerative diseases: a cross-sectional study. J. Alzheimers Dis. 59, 1327-1334 (2017).

37. Merluzzi, A. P. et al. Neurodegeneration, synaptic dysfunction, and gliosis are phenotypic of Alzheimer dementia. Neurology 91, e436-e443 (2018).

38. Pereira, J. B., Westman, E. \& Hansson, O., Alzheimer's Disease Neuroimaging Initiative. Association between cerebrospinal fluid and plasma neurodegeneration biomarkers with brain atrophy in Alzheimer's disease. Neurobiol. Aging 58, 14-29 (2017).

39. Portelius, E. et al. Cerebrospinal fluid neurogranin concentrate ion in neurodegeneration: relation to clinical phenotypes and neuropathology. Acta Neuropathol. 136, 363-376 (2018).

40. Portelius, E. et al. Cerebrospinal fluid neurogranin: relation to cognition and neurodegeneration in Alzheimer's disease. Brain 138, 3373-3385 (2015).
41. Sanfilippo, C., Forlenza, O., Zetterberg, H. \& Blennow, K. Increased neurogranin concentrations in cerebrospinal fluid of Alzheimer's disease and in mild cognitive impairment due to AD. J. Neural Transm. 123, 1443-1447 (2016).

42. Sun, X. et al. APOE $\varepsilon 4$ carriers may undergo synaptic damage conferring risk of Alzheimer's disease. Alzheimers Dement. 12, 1159-1166 (2016).

43. Sutphen, C. L. et al. Longitudinal decreases in multiple cerebrospinal fluid biomarkers of neuronal injury in symptomatic late onset Alzheimer's disease. Alzheimers Dement. 14, 869-879 (2018).

44. Tarawneh, R. et al. Diagnostic and prognostic utility of the synaptic marker neurogranin in Alzheimer disease. JAMA Neurol. 73, 561-571 (2016).

45. Vogt, N. M. et al. The gut microbiota-derived metabolite trimethylamine Noxide is elevated in Alzheimer's disease. Alzheimers Res. Ther. 10, 124 (2018).

46. Wang, L., Alzheimer's Disease Neuroimaging Initiative. Association of cerebrospinal fluid Neurogranin with Alzheimer's disease. Aging Clin. Exp. Res. 31, 185-191 (2019).

47. Wellington, $\mathrm{H}$. et al. Increased CSF neurogranin concentration is specific to Alzheimer disease. Neurology 86, 829-835 (2016).

48. Wellington, $\mathrm{H}$. et al. CSF neurogranin or tau distinguish typical and atypical Alzheimer disease. Ann. Clin. Transl. Neurol. 5, 162-171 (2018).

49. Winston, C. N. et al. Prediction of conversion from mild cognitive impairment to dementia with neuronally derived blood exosome protein profile. Alzheimers Dement. 3, 63-72 (2016).

50. Winston, C. N., Goetzl, E. J., Baker, L. D., Vitiello, M. V. \& Rissman, R. A. Growth hormone-releasing hormone modulation of neuronal exosome biomarkers in mild cognitive impairment. J. Alzheimers Dis. 66, 971-981 (2018).

51. Abner, E. L., Jicha, G. A., Shaw, L. M., Trojanowski, J. Q. \& Goetzl, E. J. Plasma neuronal exosomal levels of Alzheimer's disease biomarkers in normal aging. Ann. Clin. Transl. Neurol. 3, 399-403 (2016).

52. Reddy, P. H. et al. Differential loss of synaptic proteins in Alzheimer's disease: implications for synaptic dysfunction. J. Alzheimers Dis. 7, 103-117 (2005).

53. Ritchie, C. et al. Plasma and cerebrospinal fluid amyloid beta for the diagnosis of Alzheimer's disease dementia and other dementias in people with mild cognitive impairment (MCl). Cochrane Database Syst. Rev. 6, CD008782 (2014).

54. Ritchie, C. et al. CSF tau and the CSF tau/ABeta ratio for the diagnosis of Alzheimer's disease dementia and other dementias in people with mild cognitive impairment (MCl). Cochrane Database Syst. Rev. 3, CD010803 (2017).

55. Gouras, G. K., Olsson, T. T. \& Hansson, O. ß-amyloid peptides and amyloid plaques in Alzheimer's disease. Neurotherapeutics 12, 3-11 (2015).

56. lqbal, K., Liu, F. \& Gong, C. Tau and neurodegenerative disease: the story so far. Nat. Rev. Neurol. 12, 15-27 (2015).

57. Wildsmith, K. R. et al. Identification of longitudinally dynamic biomarkers in Alzheimer's disease cerebrospinal fluid by targeted proteomics. Mol. Neurodegener. 9, 22 (2014).

58. Casaletto, K. B. et al. Neurogranin, a synaptic protein, is associated with memory independent of Alzheimer biomarkers. Neurology 89, 1782-1788 (2017).

59. Molinuevo, J. L. et al. The clinical use of cerebrospinal fluid biomarker testing for Alzheimer's disease diagnosis: a consensus paper from the Alzheimer's Biomarkers Standardization Initiative. Alzheimers Dement. 10, 808-817 (2014).

60. Scheff, S. W., Price, D. A., Schmitt, F. A., DeKosky, S. T. \& Mufson, E. J. Synaptic alterations in $\mathrm{CA} 1$ in mild Alzheimer disease and mild cognitive impairment. Neurology 68, 1501-1508 (2007).

61. Hoffman, L., Chandrasekar, A., Wang, X., Putkey, J. A. \& Waxham, M. N. Neurogranin alters the structure and calcium binding properties of calmodulin. J. Biol. Chem. 289, 14644-14655 (2014).

62. Kvartsberg, $\mathrm{H}$. et al. The intact postsynaptic protein neurogranin is reduced in brain tissue from patients with familial and sporadic Alzheimer's disease. Acta Neuropathol. 137, 89-102 (2019).

63. Mavroudis, I. A., Petridis, F., Chatzikonstantinou, S. \& Kazis, D. A meta-analysis on CSF neurogranin levels for the diagnosis of Alzheimer's disease and mild cognitive impairment. Aging Clin. Exp. Res. https://doi.org/10.1007/s40520-01901326-z (2019).

64. Agliardi, C. \& Clerici, M. Blood extracellular vesicles (EVs) of central nervous system origin: a window into the brain. Neural Regen. Res. 15, 55-56 (2020).

65. Jia, L. et al. Concordance between the assessment of Aß42, T-tau, and P-T181tau in peripheral blood neuronal-derived exosomes and cerebrospinal fluid. Alzheimers Dement. 15, 1071-1080 (2019).

66. Jack, C. R. Jr. et al. NIA-AA research framework: toward a biological definition of Alzheimer's disease. Alzheimers Dement. 14, 535-562 (2018). 